\title{
AVALIAÇÃO DA EFICÁCIA DO GERENCIAMENTO COSTEIRO INTEGRADO UTILIZANDO AHP (ANALYTIC HIERACHY PROCESS) PARA A ILHA DE ITAMARACÁ, PERNAMBUCO, BRASIL
}

\author{
EFFECTIVENESS EVALUATION OF INTEGRATED COASTAL MANAGEMENT USING AHP \\ (ANALYTIC HIERARCHY PROCESS), FOR ITAMARACÁ ISLAND, PERNAMBUCO, BRAZIL
}

\author{
Josilene Pereira LIMA ${ }^{1}$, Rodrigo Mikosz GONÇALVES ${ }^{1}$, Marcio Augusto Relon \\ SCHMIDT $^{2}$ \\ ${ }^{1}$ Universidade Federal de Pernambuco, Programa de Pós-Graduação em Ciências Geodésicas e Tecnologias da Geoinformação, \\ Recife - PE, Brasil. Emails: josilenelima2003@yahoo.com.br; rodrigo.mikosz@ufpe.br. \\ ${ }^{2}$ Universidade Federal de Uberlândia (UFU), Faculdade de Engenharia Civil, Programa de Pós-Graduação em Engenharia Civil. \\ Uberlândia - MG, Brasil. Email: marcio.schmidt@ufu.br.
}

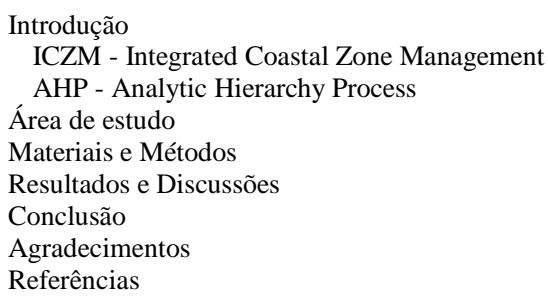

RESUMO - A gestão integrada da zona costeira (do inglês Integrated Coastal Zone Management - ICZM) envolve uma abordagem integrada de elementos naturais e antrópicos e deve ser organizada para minimizar os conflitos e utilizar os recursos de forma sustentável. A partir da definição sobre ICZM e demais legislações brasileiras foram identificadas 11 variáveis para fazer parte de um modelo de avaliação da eficácia do gerenciamento costeiro integrado utilizando a análise multicritério ponderadas através do método Analytic Hierarchy Process - AHP, considerando critérios sociais, econômicos e ambientais. O resultado deste modelo representa um valor numérico, com variação entre 0,0 e 1,0, no qual a medida que o valor aumenta melhores são as condições associadas a gestão costeira integrada. Assim, a aplicação deste método demonstrou ser uma importante ferramenta para diagnosticar e fornecer subsídios à tomada de decisão em ambientes costeiros.

Palavras-chave: gestão integrada da zona costeira; AHP (Analytic Hierarchy Process); sensoriamento remoto; sistema de informações geográficas; geotecnologias.

\begin{abstract}
Integrated Coastal Zone Management (ICZM) involves an integrated approach of natural and man-made elements and should be organized to minimize conflicts and use resources in a sustainably way. From the definition of ICZM and other Brazilian laws were identified 11 variables to be part of an evaluation model of integrated coastal management effectiveness using weighted multi-criteria analysis through the Analytic Hierarchy Process - AHP method, considering social, economic and environmental criteria. The result of this model is a numerical value ranging between 0.0 and 1.0 , in which as the value increases the better the conditions attached to integrated coastal management. Thus, this method has proven to be an important tool to diagnose and provide input to decision making in coastal environments.
\end{abstract}

Keywords: Integrated Coastal Zone Management (ICZM); AHP (Analytic Hierarchy Process); remote sensing; geographic information system; geotechnology.

\section{INTRODUÇÃO}

As zonas costeiras apresentam diversidade de características naturais favoráveis em termos de biodiversidade, clima, fertilidade e acessibilidade. Estas atraem diversas atividades como: lazer, turismo, residências, comércio e indústrias.

Consequentemente, há uma tendência para uso e ocupação excessiva deste território (Pinho et al., 2007).

Ao mesmo tempo a região costeira brasileira apresenta um quadro preocupante em relação à degradação ambiental, especialmente, em regiões próximas aos grandes centros urbanos (Asmus et al., 2006) onde é possível observar problemas relacionados com: erosão costeira, inundações, degradação de habitats naturais, poluição de diversas formas, entre outros (Coastlearn, 2012).

Para que um ambiente possa atender as demandas de todos os habitantes é necessário um controle e planejamento das diversas atividades da população respeitando os limites de sustentação natural. Dessa forma, é necessário estabelecer condutas para a vida harmoniosa entre os cidadãos (Cassilha \& Cassilha, 2009).

Uma ferramenta existente neste contexto é a gestão integrada da zona costeira. O ICZM, anacronimo da expressão em inglês Integrated Coastal Zone Management, é um conceito que utiliza uma abordagem integrada dos elementos naturais e antrópicos que atuam na zona 
costeira com o intuito de alcançar a sustentabilidade.

De acordo com Misdorp (2011), o ICZM envolve a avaliação global de atores (homem, biodiversidade, meio ambiente, sociedade, entre outros), estabelece objetivos, realiza o planejamento e gestão de sistemas e recursos costeiros, levando em conta os aspectos culturais, históricos assim como os interesses de uso conflitante.

A gestão costeira integrada objetiva manter, restaurar ou melhorar as características específicas de ecossistemas costeiros. Ela deve atender as necessidades para o desenvolvimento e conservação em lugares geograficamente específicos, considerando características globais, regionais ou locais como, por exemplo: uma comunidade, um estuário ou a costa de uma nação inteira (Olsen et al., 1997).

Uma importante iniciativa para gerenciar os sistemas costeiros vem sendo adotada no âmbito da União Europeia, onde é possível verificar a importância de comissões para propor legislação, políticas e programas de ação ao Parlamento Europeu e ao Conselho da União Europeia.

No que diz respeito ao ICZM é possível encontrar, por exemplo, documentos relacionados a ações estratégicas para a Europa (CEC, 2000), assim como recomendações (CEC, 2002).

No Brasil também existem legislações específicas para subsidiar o gerenciamento costeiro em função dos conflitos existentes e assim propor normas e estruturar políticas públicas destinadas à gestão costeira como é o caso da lei $n^{0}$ 7.661/88 que determina a elaboração do Plano Nacional de Gerenciamento Costeiro - PNGC. O PNGC tem como finalidade primordial, 0 estabelecimento de normas gerais, visando a gestão ambiental da Zona Costeira do País, lançando as bases para a formulação de políticas, planos e programas estaduais e municipais (Agra Filho, 2006).

Em nível estadual cita-se como exemplo a Lei de Gerenciamento Costeiro do Estado de Pernambuco sob n. ${ }^{\circ} 14.258 / 2010$, que objetiva promover e apoiar a conservação, recuperação e o controle de áreas que sejam representativas dos ecossistemas da zona costeira, assim como incentivar o desenvolvimento de atividades que respeitem as limitações e as potencialidades dos recursos ambientais.

A criação de normas e recomendações é fundamental para a gestão integrada da zona costeira, contudo torna-se fundamental verificar se tais instrumentos vêm sendo aplicados de forma eficaz e quais são suas deficiências.

A normatização do gerenciamento costeiro somente é eficaz se puder ser monitorado por elementos quantitativos, e isso pode ser feito por modelos que fazem uso de observações globais, indicadores de sucesso ou insucesso de cada uma das tarefas no processo de gerenciamento. Alguns modelos são avaliados a partir de dados globais e através de indicativos conforme a conclusão de uma determinada tarefa com sucesso ou não. A complexidade espacial e características físicas e geográficas locais para gestão costeira devem ser consideradas.

Desta forma, este trabalho teve como objetivo a elaboração de uma modelagem analítica utilizando o modelo chamado Analytic Hierachy Process (AHP), considerando aspectos socioeconômicos e ambientais, para avaliar numericamente a eficácia da gestão costeira integrada em um estudo de caso considerando a ilha de Itamaracá localizada no estado de Pernambuco, Brasil.

\section{ICZM - Integrated Coastal Zone Management}

A zona costeira pode ser caracterizada como um sistema que interliga os ambientes terrestres e marinhos, com as atividades humanas representando um dos ambientes naturais mais dinâmicos e ameaçados. Além disso, este ambiente tornou-se o principal local de interação entre as atividades humanas.

Dada à diversidade natural e à fragilidade do ambiente costeiro diante da demanda por recursos costeiros e dos impactos humanos (crescimento populacional, degradação dos hábitats, múltiplos conflitos de uso e exploração dos recursos) combinada com as mudanças climáticas globais tem motivado conflitos entre usuários. Uma melhor compreensão das inter-relações entre as variáveis naturais e socioeconômicas é essencial para a gestão sustentável da zona costeira (Fabbri, 1998).

$\mathrm{Na}$ Conferência das Nações Unidas sobre Meio Ambiente e Desenvolvimento (UNCED), 
no Rio de Janeiro, em 1992, houve a inclusão do conceito sobre Gestão Integrada da Zona Costeira com o intuito de compreender todas as atividades e recursos da zona costeira e como lidar com as questões econômicas e sociais, bem como as preocupações ambientais/ ecológicas (Post \& Lundin, 1996). Uma das principais recomendações da Agenda 21 refere-se a este tema e sua importância internacional e legitimidade política (Cummins et al., 2003).

A gestão costeira integrada pode ser definida como um processo contínuo e dinâmico para alcançar o desenvolvimento sustentável das zonas costeiras e marinhas para reduzir a vulnerabilidade das zonas costeiras e os riscos naturais aos seus habitantes, e para manter os processos ecológicos essenciais, sistemas de suporte à vida, e da diversidade biológica em áreas costeiras e marinhas (Cicin-Sain \& Knecht, 1998).

A necessidade de estabelecer um programa de gestão costeira integrada pode surgir por diversos motivos: esgotamento dos recursos costeiros e oceânicos; aumento da poluição, que ameaça a saúde pública e as atividades relacionadas ao uso da água; para aumentar os benefícios econômicos obtidos com a utilização da costa e do oceano; e o desejo de desenvolver atividades como a aquicultura marinha e a extração de petróleo em alto mar. Evidencia-se, portanto, a importância de analisar variáveis e métodos que possam quantificar os conflitos existentes no uso dos recursos costeiros.

\section{AHP - Analytic Hierarchy Process}

O método AHP tem sido empregado em diversas áreas: análise de custo/benefício, planejamento estratégico, investimentos econômicos, análise ambiental, entre outros. O AHP baseia-se na comparação de critérios pareados elencados e ordenados por especialistas. Schmidt \& Barbosa (2016) afirmam que esta técnica envolve incertezas no processo por não considerar o caráter subjetivo inerente às avaliações dos especialistas humanos nas ponderações iniciais, o que é semelhante, por exemplo, às técnicas supervisionadas em sensoriamento remoto. Portanto, é importante destacar que a AHP somente avalia a consistência da matriz de valores pareados do sistema em um contexto específico para determinar os pesos relativos e utiliza os julgamentos dos tomadores de decisão para formar uma decomposição de problemas em hierarquias (Podvezko, 2011).

A complexidade do problema é representada pelo número de níveis na hierarquia que combinam com o modelo do tomador de decisão no problema a ser resolvido (Alexander, 2012).

A aplicação do AHP pode ser dividida em três etapas:

(i) Estruturação do problema em hierarquias

O problema deve ser estruturado em níveis hierárquicos, de modo que possibilite uma avaliação pormenorizada dos diversos aspectos do problema. Uma hierarquia é uma abstração da estrutura de um sistema para estudar as interações funcionais de seus componentes e seus impactos no sistema total que também facilita o processo de raciocínio humano (Alves \& Alves, 2015).

(ii) Definição de prioridades e julgamentos

As prioridades de um critério sobre outro ou de uma alternativa sobre outra são estabelecidas através de comparações par a par fundamentadas nas observações de um especialista, que determina a importância relativa entre eles (Alves \& Alves, 2015). Estes critérios são organizados em uma matriz de decisão quadrada denominada matriz de comparação par a par, matriz dominante ou matriz de juízo. A paridade é realizada pela importância relativa de cada critério em relação aos demais.

Esta verificação da importância que o tomador de decisão expressa, baseia-se na sua experiência. Cada escolha pode ser demonstrada por uma expressão linguística, como: "A é mais importante do que $B$ " ou " $A$ tem a mesma importância de $B$ ", ou " $A$ é um pouco mais importante do que $B$ ". Estas expressões linguísticas são convertidas em valores numéricos usando a Escala Fundamental de Saaty (1990), onde essa escala de valores varia de 1 a 9 (Figura 1 ).

(iii) Matriz prioridade e consistência das comparações

Saaty (1990) permitiu algumas medidas de inconsistência (comum com o julgamento humano subjetivo) quando aplicada à lógica das preferências. Inconsistências surgem quando se comparam três itens, $A, B$, e $C$. A AHP inclui 
uma técnica para verificar a consistência das avaliações realizadas pelo tomador de decisão na construção de cada uma das matrizes de comparação de pares envolvidos no processo. A técnica baseia-se no cálculo de um índice de consistência.

\begin{tabular}{|c|c|l|}
\hline $\begin{array}{c}\text { Intensidade de } \\
\text { Importância }\end{array}$ & Definição & \multicolumn{1}{c|}{ Explicação } \\
\hline $\mathbf{1}$ & Mesma importância & As duas atividades contribuem igualmente para o objetivo \\
\hline $\mathbf{3}$ & $\begin{array}{c}\text { Importância pequena de uma } \\
\text { sobre a outra }\end{array}$ & $\begin{array}{l}\text { A experiência e o julgamento favorecem levemente uma } \\
\text { atividade em relação a outra. }\end{array}$ \\
\hline $\mathbf{5}$ & $\begin{array}{c}\text { Importância grande ou } \\
\text { essencial }\end{array}$ & $\begin{array}{l}\text { A experiência e o julgamento favorecem fortemente uma } \\
\text { atividade em relação a outra. }\end{array}$ \\
\hline $\mathbf{7}$ & $\begin{array}{c}\text { Importância muito grande ou } \\
\text { demonstrada }\end{array}$ & $\begin{array}{l}\text { Uma atividade é muito fortemente favorecida em relação a } \\
\text { outra; sua dominação de importância é demonstrada na prática }\end{array}$ \\
\hline $\mathbf{9}$ & $\begin{array}{c}\text { Importância absoluta } \\
\text { Quando se procura uma condição de compromisso entre duas } \\
\text { definiços. }\end{array}$ \\
\hline $\mathbf{2 , 6 , 8}$ & $\begin{array}{c}\text { Valores intermediários entre } \\
\text { os valores adjacentes }\end{array}$ & Quando se procura uma condição entre duas definições \\
\hline
\end{tabular}

Figura 1 - Escala Fundamental de Saaty para julgamentos comparativos. Fonte: Saaty (1990).

\section{ÁREA DE ESTUDO}

O município da Ilha de Itamaracá está situado no litoral norte de Pernambuco e faz parte da Região Metropolitana de Recife, localizado a cerca de $40 \mathrm{~km}$ da capital pernambucana.
Limitando-se a norte com Goiana, a sul com Igarassu, a leste com Oceano Atlântico, e a oeste com Itapissuma (Figura 2).

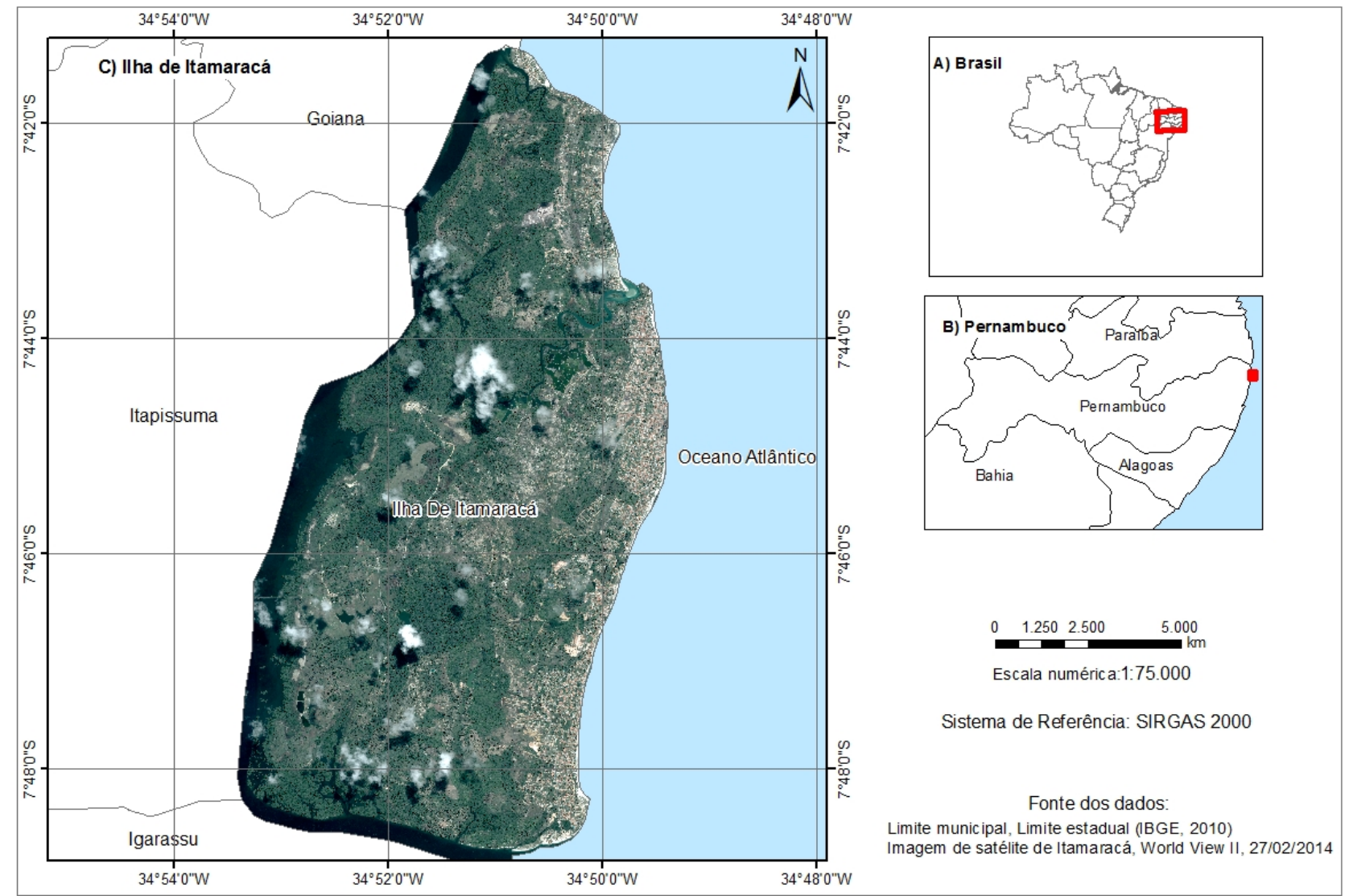

Figura 2 - Mapa de localização do município da Ilha de Itamaracá. Onde: A) representa o mapa do Brasil localizando o Estado de Pernambuco, B) o Estado de Pernambuco e C) a imagem de satélite da Ilha de Itamaracá. Fonte da imagem de satélite recursos do projeto Universal/CNPq14/2012, nº 482224/2012.

O município de Itamaracá está inserido na unidade geoambiental da Baixada Litorânea, com relevo formado pelas Áreas Arenosas Litorâneas, onde se incluem as restingas, as dunas e os mangues. A vegetação é basicamente formada por Florestas Perenifólia de Restinga. O clima é 
do tipo Tropical Chuvoso com precipitação média anual é de $1.867 \mathrm{~mm}$.

Os principais rios são os Paripe e o Jaguaribe (CPRM, 2005). O município possui algumas reservas ecológicas definidas pela Lei Estadual $\mathrm{n}^{\mathrm{o}}$. 9.989/87, que abrange as seguintes matas: Lanço dos Cações; Santa Cruz; Jaguaribe; Engenho Macaxeira; Engenho São João; Amparo.

Há também reservas biológicas constituídas pelas áreas estuarinas definidas na Lei Estadual $\mathrm{n}^{\circ}$. 9.931/86, abrangendo: a área estuarina do rio Jaguaribe e a área estuarina do canal de Santa
Cruz.A ilha de Itamaracá possui uma área de 66 $\mathrm{km}^{2}$ e 21.884 habitantes (IBGE, 2010), uma população urbana de 16.993 e rural de 4.891 habitantes com taxa de urbanização de 77,65\%.

Pela análise destes dados do Censo Demográfico, observa-se que a Ilha de Itamaracá foi marcada pelo crescimento populacional com um aumento de aproximadamente $200 \%$ entre os anos de 1970 e 2010.

O Município tem uma população flutuante significativa, devido à quantidade de residências destinadas ao veraneio.

\section{MATERIAIS E MÉTODOS}

A primeira etapa foi a obtenção dos dados a serem usados na análise.

Como fonte de informações para a modelagem proposta foram selecionados: dados socioeconômicos obtidos do censo demográfico do IBGE (Instituto Brasileiro de Geografia e Estatística) para os anos de 2000 e 2010; os limites das unidades de conservação; uma ortofotocarta do ano de 1989, imagens de satélites artificiais QuickBird referente ao ano de 2005 e WorldView-2 referente ao ano de 2014; informações vetoriais hidrográficas e dados do Índice de Desenvolvimento Humano Municipal - IDH-M (O IDH-M brasileiro considera as mesmas três dimensões do IDH Global - longevidade, educação e renda, adequando a metodologia global ao contexto brasileiro e à disponibilidade de indicadores nacionais) disponibilizado no Atlas de Desenvolvimento Humano elaborado pelo Programa das Nações Unidas para o Desenvolvimento - PNUD, Instituto de Pesquisa Econômica Aplicada - IPEA e Fundação João Pinheiro.

A segunda etapa foi a definição das 11 variáveis descritivas do problema e que foram utilizadas como dados ponderados de entrada no modelo AHP sendo elas descritas a seguir:

a) Coleta de resíduos sólidos - razão entre o total de domicílios particulares permanentes com lixo coletado por serviço de limpeza e o total de domicílios particulares permanentes;

b) Sistema de abastecimento de água - razão entre 0 total de domicílios particulares permanentes com abastecimento de água da rede geral e o total de domicílios particulares permanentes;

c) Acesso a esgotamento sanitário - razão entre o total de domicílios particulares permanentes com banheiro de uso exclusivo dos moradores ou sanitário e esgotamento sanitário via rede geral de esgoto ou pluvial e o total de domicílios particulares permanentes com banheiro de uso exclusivo dos moradores ou sanitário.

d) IDH-M Longevidade - não podem ser obtidos diretamente das informações dos Censos Demográficos, recorrendo-se, então, a técnicas indiretas para sua obtenção, pois não é conhecido o padrão de mortalidade de cada população.

e) IDH-M Educação - é uma composição de indicadores de escolaridade da população adulta e de fluxo escolar da população jovem.

f) IDH-M Renda - o indicador corresponde à razão entre o somatório de todos os rendimentos de todos os indivíduos residentes no lugar de referência, recebidos no mês anterior à data do Censo, e o número total desses indivíduos;

g) Índice de Gini - numericamente, varia de 0 a 1 , sendo que 0 representa a situação de total igualdade, ou seja, todos têm a mesma renda, e o valor 1 significa completa desigualdade de renda, ou seja, se uma só pessoa detém toda a renda do lugar;

h) PIB per capita - Razão entre PIB per capita do município e o PIB per capita do Estado de Pernambuco.

i) Áreas preservadas - 0 indicador foi calculado pela relação das áreas de preservação em concordância com a legislação, para tal foram utilizados os limites das áreas de preservação mapeadas pelo órgão estadual responsável a Agência Estadual de Meio Ambiente e Recursos Hídricos (CPRH) que 
estão classificadas em: reservas ecológicas definidas na Lei Estadual $\mathrm{n}^{\circ}$. 13.539/08; reservas biológicas constituídas pelas áreas estuarinas definidas na Lei Estadual $\mathrm{n}^{\circ}$. 9.931/86; e áreas de preservação permanente calculada com base no código florestal e na resolução CONAMA 303 de 2002.

j) Erosão Costeira - a fim de configurar e mapear a variável erosão costeira foi feito uma estimativa, fornecendo um valor numérico e para isso considerou-se a escala de 0 a 1 a qual o valor numérico igual a 1 indica um alto grau de processos erosivos até 0 valor 0 , considerando a linha de costa como estável. O cálculo do indicador é realizado pelo valor médio atribuído conforme a razão entre a faixa costeira de retrogradação e suas variações de valores pela faixa total. $\mathrm{O}$ indicativo para delinear a linha de costa e identificá-la nas imagens de satélite artificial considerado foi o alcance máximo da maré para o determinado instante de tempo da tomada da imagem digital, normalmente, fotointerpretado como a linha de vegetação e ou em casos urbanizados com problemas erosivos no limite das construções.

k) População urbana - a população urbana foi calculada pela razão entre a população da área urbana e a população total do município.

\begin{tabular}{|l|c|c|}
\hline \multicolumn{1}{|c|}{ Variável } & $\mathbf{2 0 0 0}$ & $\mathbf{2 0 1 0}$ \\
\hline IDH-M Longevidade & 0,761 & 0,809 \\
\hline IDH-M Educação & 0,410 & 0,548 \\
\hline Coleta de resíduos sólidos & 0,166 & 0,669 \\
\hline Sistema de abastecimento de água & 0,503 & 0,756 \\
\hline Acesso a esgotamento sanitário & 0,003 & 0,011 \\
\hline IDH-M Renda & 0,590 & 0,627 \\
\hline PIB Per capita & 0,001 & 0,001 \\
\hline Índice de Gini & 0,410 & 0,400 \\
\hline Áreas preservadas & 0,880 & 0,874 \\
\hline Proteção da linha de costa (Erosão costeira) & 0,839 & 0,776 \\
\hline População Área Urbana & 0,185 & 0,223 \\
\hline
\end{tabular}

Figura 3 - Valores numéricos dos indicadores.

Ressalta-se que o cálculo da variável população área urbana foi calculada pela relação inversa da quantidade populacional na área urbana, ou seja, quanto maior for a quantidade populacional na área urbana menor a eficácia para o ICZM. Desta forma, o cálculo do indicador foi calculado: (1 - População Área Urbana).

O Índice de Gini também seguiu a mesma diretriz, pois numericamente, varia de 0 a 1 . Em seguida, a estrutura hierárquica foi dividida em três níveis definidos conforme a Figura 4. Na classe 1, encontra-se o objetivo do modelo, que é avaliar a eficácia do ICZM.

Os critérios estão definidos na classe 2. $\mathrm{Na}$ terceira classe hierárquica estão as 11 variáveis consideradas para o problema.

Para a dimensão social procurou-se agrupar e identificar os principais aspectos relacionados à infraestrutura e as condições sociais compostos por cinco variáveis: coleta de resíduos sólidos, acesso ao sistema de abastecimento de água, acesso ao sistema de esgotamento sanitário, IDH-
M longevidade e IDH-M educação.

Para a dimensão econômica foram incluídas como variáveis: a IDH-M Renda, o índice de Gini e o PIB per capita.

Para dimensão ambiental foram utilizadas as áreas preservadas, a densidade urbana e a variável nomeada como erosão costeira, ao considerar que as atividades antrópicas podem causar degradação ambiental quando desrespeitam os limites naturais do ecossistema.

A etapa mais sensível é a atribuição dos pesos. Esta consistiu em estabelecer o grau de importância entre as variáveis. A fim de obter as prioridades em todos os critérios foi aplicado um questionário para um especialista com mais de 20 anos de experiência que coordena a parte de gerenciamento costeiro no Estado de Pernambuco.

De acordo com as respostas nota-se que no nível 1 o gestor atribuiu importância maior aos critérios sociais e no econômico atribuiu mesma prioridade. Em contrapartida, o critério ambiental teve o menor valor 


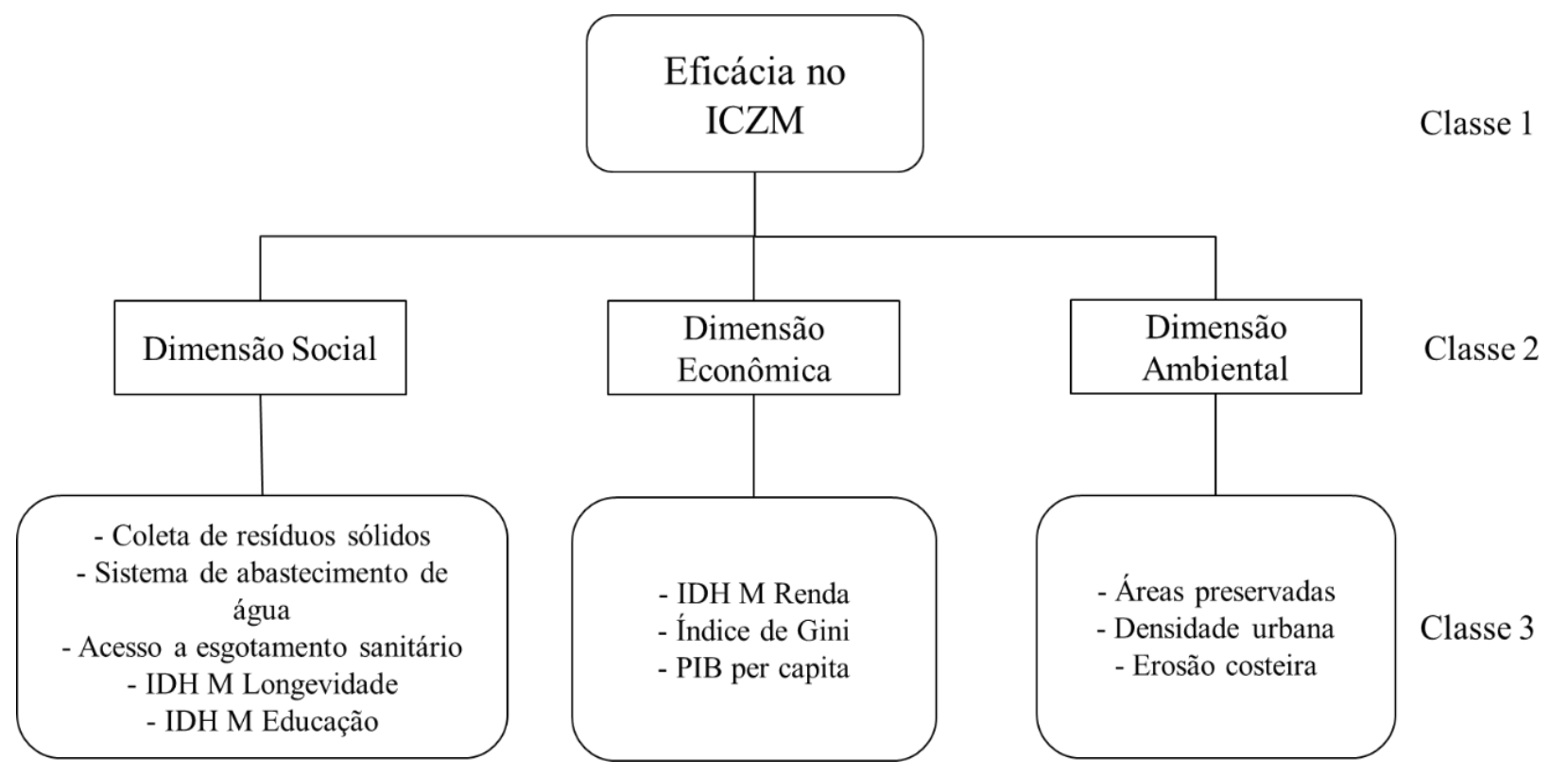

Figura 4. Estrutura hierárquica das variáveis.

No nível 2 dentre as variáveis do critério social o sistema de esgotamento sanitário teve maior prioridade para garantir a eficácia do gerenciamento costeiro, em seguida o IDH-M longevidade teve o segundo valor na prioridade.
As variáveis resíduos sólidos e IDH-M educação atribui-se os mesmos valores e por último com menor prioridade ficou a variável abastecimento de água. Para as variáveis do critério econômico e ambiental o gestor atribuiu igual prioridade (Figura 5).

\begin{tabular}{|c|c|c|c|}
\hline Nível 1 & Pesos & Variáveis - Nível 2 & Pesos \\
\hline \multirow{6}{*}{ Critério Social } & \multirow{6}{*}{0,455} & Sistema de abastecimento de água & 0,074 \\
\hline & & Coleta de Resíduos sólidos & 0,137 \\
\hline & & Acesso a esgotamento sanitário & 0,404 \\
\hline & & IDH-M Longevidade & 0,248 \\
\hline & & IDH-M Educação & 0,137 \\
\hline & & Total & 1 \\
\hline \multirow{4}{*}{ Critério Econômico } & \multirow{4}{*}{0,455} & IDH-M Renda & 0,333 \\
\hline & & PIB Per capita & 0,333 \\
\hline & & Índice de Gini & 0,333 \\
\hline & & Total & 1 \\
\hline \multirow{4}{*}{ Critério Ambiental } & \multirow{4}{*}{0,090} & Áreas preservadas & 0,333 \\
\hline & & Proteção da linha de costa (Erosão costeira) & 0,333 \\
\hline & & População Área Urbana & 0,333 \\
\hline & & Total & 1 \\
\hline Total & 1 & & \\
\hline
\end{tabular}

Figura 5 - Pesos das variáveis.

Conforme a estruturação hierárquica definida na Figura 2, as variáveis utilizadas na Classe 2 e na Classe 3 foram comparadas "par a par”, de acordo com as importâncias estabelecidas pelo gestor.
Com base nos valores dos indicadores das variáveis e nas prioridades atribuída a cada critério da Classe 3 calculou-se os indicadores da classe 2: dimensão social, dimensão econômica e dimensão ambiental a partir da equação (1). 


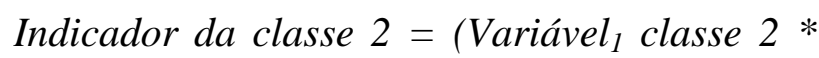
${\text { Peso } \text { Variável }_{1}+\text { Variável }_{2} \text { classe }_{2} * \text { Peso }}$ Variável $_{2}+$ Variável $_{N}$ classe $2 *$ Peso Variável $_{N}$ )

A modelagem da eficácia do ICZM é determinada pelo cálculo de cada indicador da classe 2, conforme a equação (2) e a prioridade de cada indicador.

Eficácia ICZM $=\left(D S^{*}\right.$ Peso $\mathrm{DS}^{+} \mathrm{DE}^{*}$ Peso $D E$ $+D A *$ Peso $\left._{D A}\right)$

Sendo DS = Dimensão Social;

$D E=$ Dimensão Econômica;

\section{$D A=$ Dimensão Ambiental.}

A fim de comparar os valores adquiridos com os indicadores do município da Ilha de Itamaracá foram também calculados os indicadores máximos e mínimos para todos os municípios da zona costeira do Estado de Pernambuco (Goiana, Itaquitinga, Itapissuma, Araçoiaba, Igarassu, Abreu e Lima, Paulista, Camaragibe, Olinda, Recife, São Lourenço da Mata, Moreno, Jaboatão dos Guararapes, Cabo de Santo Agostinho, Ipojuca, Sirinhaém, Rio Formoso, Tamandaré, Barreiros, São José da Coroa Grande) tendo o ano de 2010 como referência.

\section{RESULTADOS E DISCUSSÕES}

Tendo como base a dimensão social, econômica e ambiental foi construída a figura 6 que apresenta a Classe 2 e suas subdivisões considerando as dimensões da Classe 3 com os

respectivos valores máximos e mínimos para cada variável dos municípios da zona costeira do Estado de Pernambuco.

\begin{tabular}{|c|l|c|c|}
\hline Dimensão & \multicolumn{1}{|c|}{ Variável } & Valor Mínimo & Valor Máximo \\
\hline \multirow{5}{*}{ Social } & Coleta de resíduos sólidos & 0,470 & 0,947 \\
\cline { 2 - 4 } & Sistema de abastecimento de água & 0,576 & 0,932 \\
\cline { 2 - 4 } & Acesso a esgotamento sanitário & 0,009 & 0,542 \\
\cline { 2 - 4 } & IDH-M Longevidade & 0,715 & 0,836 \\
\cline { 2 - 4 } & IDH-M Educação & 0,479 & 0,703 \\
\hline \multirow{3}{*}{ Econômica } & IDH-M Renda & 0,544 & 0,798 \\
\cline { 2 - 4 } & PIB Per capita & 0,000 & 0,310 \\
\cline { 2 - 4 } & Índice de Gini & 0,320 & 0,560 \\
\hline \multirow{3}{*}{ Ambiental } & Áreas preservadas & 0,000 & 0,633 \\
\cline { 2 - 4 } & Proteção da linha de costa (Erosão costeira) & 0,300 & 0,700 \\
\cline { 2 - 4 } & População Área Urbana & 0,000 & 0,470 \\
\hline
\end{tabular}

Figura 6 - Valores dos indicadores máximo e mínimo para os municípios da zona costeira de Pernambuco.

Com os valores numéricos da figura 5 , foram feitas estimativas para estabelecer qual seria a variação da eficácia do ICZM considerando os valores máximos e mínimos como parâmetros de entrada no modelo considerando as equações (1) e (2). Como resultados numéricos a partir deste cálculo foram obtidos o valor máximo de 0,650 e o valor mínimo de 0,279. Apresentando uma variação na dimensão social de 0,450 a 0,792 , na dimensão econômica de 0,288 a 0,556 e uma variação na dimensão ambiental de 0,100 a 0,601, conforme representação gráfica representada na Figura 7.

Neste cálculo da eficácia do ICZM máxima é possível observar algumas variáveis que influenciam mais no modelo como é o caso da variável coleta de resíduos sólidos e abastecimento de água, onde assumiram valores acima de 90\% em alguns municípios. Em relação aos valores mínimos (Figura 6) da eficácia do ICZM a dimensão ambiental apresentou valores baixos nos municípios de Goiana, Itaquitinga, Araçoiaba, Itapissuma, Sirinhaém, Rio Formoso, Barreiros, São José da Coroa Grande devido a inexistência de áreas destinadas para proteção ambiental.

A população urbana apresenta valores nulos devido ao forte adensamento populacional quando a população total está inserida em área urbana, como o caso dos municípios de Recife, Paulista, Olinda, Jaboatão dos Guararapes e Camaragibe. 


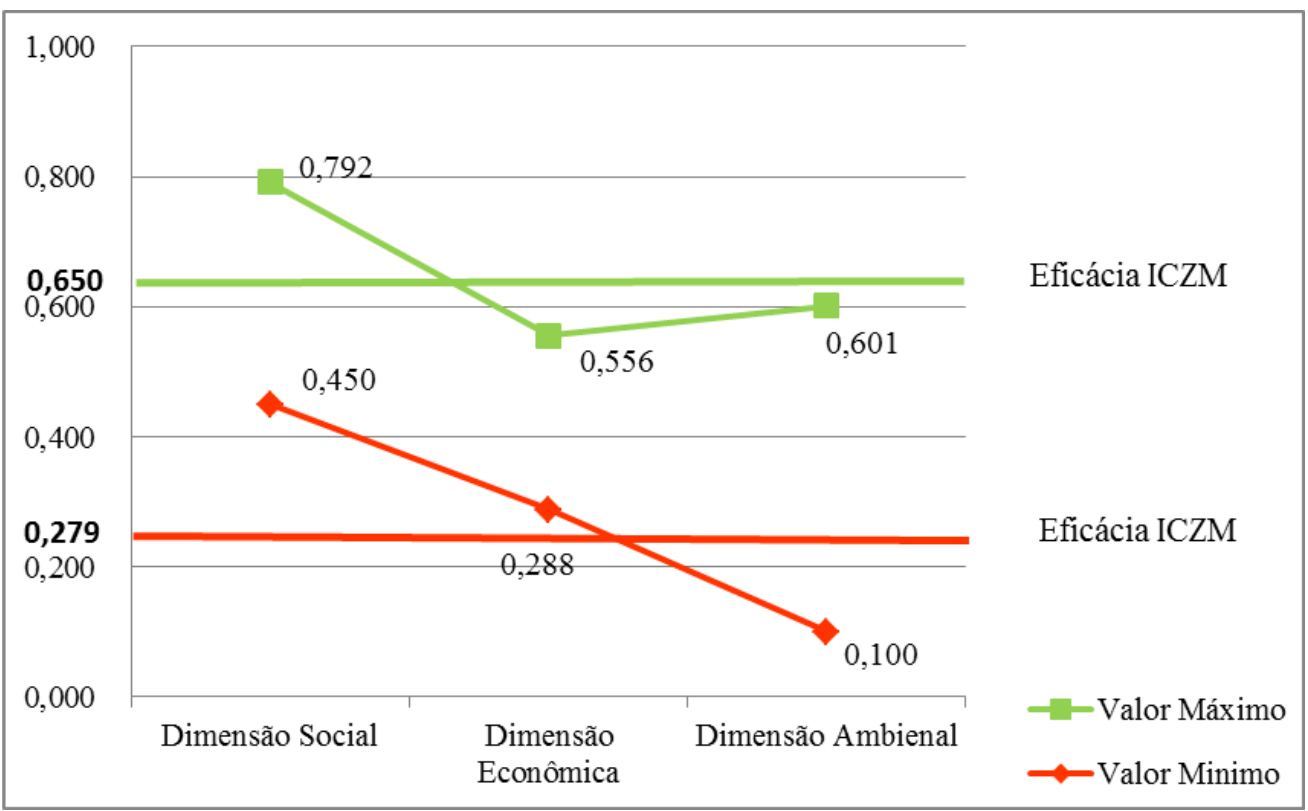

Figura 7 - Valor da eficácia máxima e mínima para os municípios da zona costeira de Pernambuco.

Através desta análise foi possível perceber discrepâncias nos valores das variáveis ao longo da zona costeira de Pernambuco. Enquanto o município de Tamandaré assume valor no índice de 0,479 para o IDH-M educação, o município de Paulista apresenta uma perspectiva otimista assumindo o valor de 0,703 . Outro exemplo é a variação do IDH-M renda que tem o município de Araçoiaba apresentando valor 0,544 e o município de Recife apresentando com valores de 0,798.

Esta variação revela diferentes níveis de desenvolvimento entre os municípios e consequentemente influenciam nas equações (1) e (2).

Na análise comparativa da eficácia do ICZM na Ilha de Itamaracá para o ano 2000 encontrou-se o valor de 0,348 (34\%). A comparação através de uma simples análise linear deste valor com os valores máximos e mínimos e os demais valores dos municípios da zona costeira de Pernambuco foi possível verificar que o resultado encontrado está abaixo de $50 \%$ indicando necessidade de melhoria tanto nos setores sociais como econômicos.

Com relação ao ano de 2010 o valor da eficácia foi de 0,407 (40\%) apresentando uma melhora em comparação com o ano de 2000, porém continua com um indicativo abaixo de $50 \%$, apesar de melhorias apresentadas nos indicadores sociais e econômicos (Figura 8).

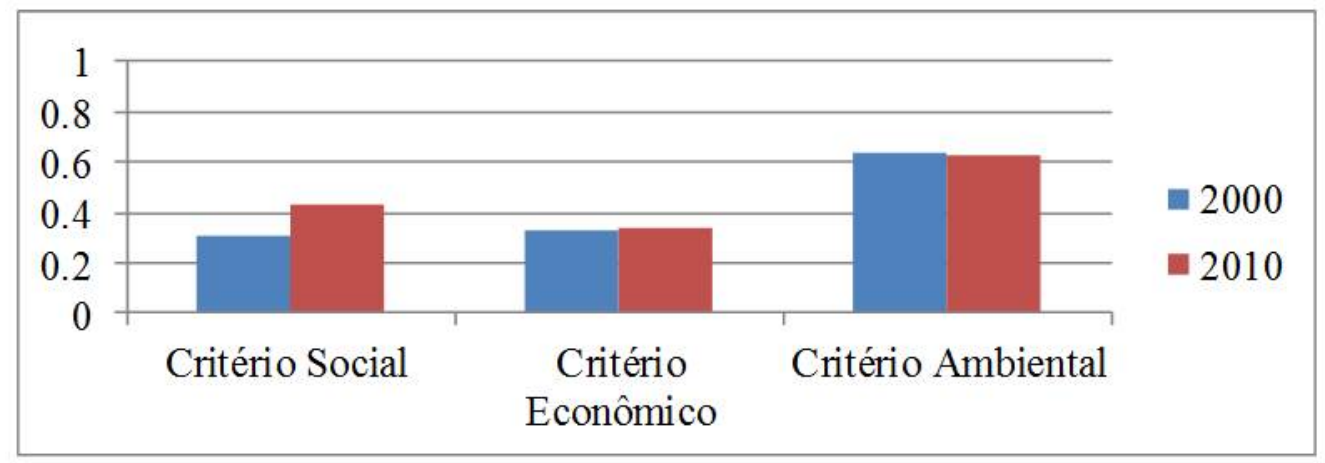

Figura 8 - Eficácia ICZM para o ano 2000 e 2010 para a Ilha de Itamaracá.

Ao comparar os valores dos critérios entre os dois anos (2000 e 2010) conclui-se que o maior índice está no critério ambiental devido aos valores dos indicadores apresentados nas variáveis áreas preservadas e proteção da linha de costa (erosão costeira).

O critério econômico apresentou pequena variação em seu crescimento, aumentando 3,79\%.

O critério social foi o único com um crescimento expressivo de $28,3 \%$, entretanto, a variável sistema de esgotamento sanitário não influenciou no aumento do índice porque no período de 10 anos o valor deste indicador teve um aumento de apenas $0,008 \%$, praticamente 
desconsiderável.

Durante este intervalo verificou-se a aprovação do plano diretor do município para o ano de 2007 trazendo orientações sobre o ordenamento do território urbano, em que há um enfoque destinado ao saneamento ambiental que considera medidas para preservar o meio ambiente através de ações de abastecimento de água potável, de esgotamento sanitário, de coleta e disposição de resíduos sólidos.

No entanto, apesar das várias diretrizes estabelecidas para o sistema de esgotamento sanitário, poucos foram os avanços para estas ações.

Quanto ao critério ambiental manteve-se certa estabilidade, mesmo com uma pequena diminuição da faixa de proteção da linha de costa, as áreas especiais de proteção (reservas ecológicas e reservas biológicas) definidas por lei estadual, e as áreas de preservação permanente foram consolidadas. Além, da faixa de proteção da orla que consiste numa área de 50 metros de proteção, no qual são definidos critérios para uso e ocupação.

Como ação voltada ao ordenamento dos espaços litorâneos tem-se o projeto orla aprovado no ano de 2010, que apresenta o diagnostico dos principais conflitos ambientais, sociais e econômicos.

O projeto orla em sua caracterização não analisou os dados referente ao censo do IBGE 2010, mas já apontava a necessidade de melhorias em aspectos relacionados a infraestrutura como o sistema de esgotamento sanitário, serviços de abastecimento de água e a coleta de resíduos.

Ao comparar os valores de eficácia de ICZM para os anos de 2000 e 2010 com os valores considerados padrões para verificar a eficácia tendo em vista as variáveis adotadas e os municípios da zona costeira de Pernambuco, verificou-se que o índice está abaixo do valor máximo almejado $(0,650)$.

\section{CONCLUSÕES}

Neste trabalho foi elaborado um modelo para analisar a eficácia do ICZM, em que a partir da definição sobre ICZM e demais legislações brasileiras foram identificadas e selecionadas algumas variáveis para atuarem no modelo.

As variáveis foram organizadas numa estrutura hierárquica de acordo com o método AHP, no qual foram atribuídos os pesos por meio de um questionário aplicado a um especialista em Gestão Costeira do Estado de Pernambuco.

Pela utilização deste questionário foi desenvolvida a comparação par a par, e a aplicação da fórmula analítica que representa a saída do modelo da eficácia do ICZM em valores numéricos numa escala de 0 a 1 , onde quanto maior a nota mais eficaz o gerenciamento costeiro integrado.

Para analisar os resultados numéricos da eficácia do ICZM no município da Ilha de Itamaracá, foram calculados limiares mínimo e máximo tendo como referência os municípios pertencentes à zona costeira de Pernambuco.

Os dados estruturados pelo método AHP foram analisados para os anos 2000 e 2010 através do modelo analítico considerando os pesos e prioridades definidas pelo especialista.

O valor numérico da eficácia encontrado para o ano de 2000 foi de 0,348 abaixo de 50\% na comparação para um valor ideal considerando as características dos demais municípios do Estado de Pernambuco o que indica necessidade de melhoria nos indicadores sociais e econômicos com valores obtidos no limiar máximo de 0,650 e o limiar mínimo de 0,279. Para o ano de 2010 verificou-se que houve melhoria, quanto aos indicadores sociais e econômicos, no entanto, o valor obtido pelo modelo analítico foi de 0,407 continuando ainda abaixo de $50 \%$.

Comparando as variáveis temporais (2000 e 2010) para o estudo de caso considerando o município da Ilha de Itamaracá foi possível perceber:

(a) Em relação aos valores nas variáveis sociais houve aumento na proporção de domicílios com sistema de abastecimento de água, aumentando em alguns setores para 81\% a concentração de domicílios;

(b) Na coleta de resíduos sólidos houve um aumento de $70 \%$ na quantidade de domicílios atendidos.

No entanto, ainda se observa: (i) carência no sistema de esgotamento sanitário, pois houve pouco avanço na implantação dessa infraestrutura, a totalidade do território não atinge $1 \%$ com sistema de esgotamento tanto para o ano 
de 2000 quanto para o ano de 2010; (ii) os valores das variáveis IDH-M Longevidade, IDH-M Educação e IDH-M Renda tiveram crescimento no seu valor, tendo o IDH-M Educação um aumento no seu valor de 25,18\%.

Em contrapartida o IDH-M Longevidade apresentou valores altos para os dois períodos analisados; (iii) quanto as variáveis ambientais as áreas preservadas tiveram pouca alteração no valor do indicador.

A análise temporal das imagens revelou diminuição na faixa de proteção da linha de costa, de $84 \%$ para $78 \%$.

Os resultados obtidos com a aplicação da metodologia AHP ao processo decisório na análise da eficácia do gerenciamento costeiro demonstram a utilidade dos métodos multicritérios de apoio à decisão.

Com o intuito de melhorar a qualidade do modelo é possível em trabalhos futuros inserir novas variáveis de entrada, assim como testar outros métodos de avaliação multicritério.

Considerando a característica insular do município de Itamaracá é importante integrar perspectivas de conservação e desenvolvimento de forma a haver equilíbrio, exigindo abordagens específicas para planejamento e gestão.

\section{AGRADECIMENTOS}

Os autores gostariam de agradecer ao suporte dos seguintes projetos: Universal/CNPq 14/2012, no do Proc.: 482224/2012-6 e PQ/nível 2/CNPq nº do Proc. 310412/2015 - 3/PQ.

\section{REFERÊNCIAS}

AGRA FILHO, S. S. A gestão ambiental da zona costeira no Brasil. Os desafios atuais. Revista de Gestão Costeira Integrada para países de língua portuguesa, n. 5, ano. 4, p. 23-24, 2006. Disponível em: <http://www.aprh. pt/rgci/pdf/RGCI_5.pdf> Acesso em: 08 de jan. de 2014.

ALEXANDER, $\bar{M}$. Decision-Making using the Analytic Hierarchy Process (AHP) and SAS/IML. SESUG 2012: The Proceedings of the SouthEast SAS Users Group, Durham, NC, p. 1-12, 2012. Disponível em: < http://analytics.ncsu. edu/sesug/2012/SD-04.pdf>. Acesso em: 10 de jan. de 2015.

ALVES, J. R. X. \& ALVES, J. M. Definição de localidade para instalação industrial com o apoio do método de análise hierárquica (AHP). Production Journal, v. 25, n. 1, p. 13-26, 2015.

ASMUS, M. L.; KITZMANN, D.; LAYDNER, C.; TAGLIANI, C. R. A. Gestão Costeira no Brasil: instrumentos, fragilidades e potencialidades. Revista de Gestão Costeira Integrada para países de língua portuguesa, n. 5, ano. 4, p. 52-57, 2006. Disponível em: <http://www.aprh.pt/rgci/pdf/RGCI_5.pdf> Acesso em: 08 de jan. de 2014.

CASSILHA, G. A. \& CASSILHA, S. A. Planejamento Urbano e Meio Ambiente. Curitiba: IESDE Brasil S. A., 2009.

CICIN-SAIN, B. \& KNECHT, R. Integrated coastal and ocean management: concepts and practices. Washington, DC - Island Press, 543 p., 1998.

COASTLEARN, A multimedia distance training package. Principles of ICZM, 2012. An initiative of Coastal \& Marine Union - EUCC (Leiden) and the EUCC Mediterranean Centre (Barcelona). Disponível em: http://www.coastlearn. org/intro/introduction.html. Acesso em: 03 de jan. de 2014.

CUMMINS, V.; O MAHONY, C.; CONNOLLY, N. Review of integrated coastal zone management $\&$ principals of best practice, 2003. Disponível em: http://www.heritagecouncil. ie/fileadmin/user_upload/Publications/Marine/coastal_zone_r eview.pdf. Acesso em: 04 de jan. de 2014.

CEC. Commission of the European Communities. Integrated Coastal Zone Management: a strategy for Europe. Communication from the Commission to the Council and the European Parliament, Brussels, COM 547 final, 27 p., 2000. Disponível em: http://eur-lex.europa.eu/legal-content/EN /TXT/ PDF/?uri=CELEX:52000DC0547\&from=EN. Acesso em: 17 de nov. de 2016.

CEC. Commission of the European Communities. The Implementation of Integrated Coastal Zone Management in
Europe. Council recommendation of the European Parliament and of the Council of 30 May, 4 p., 2002. Disponível em: http://eur-lex.europa.eu/legal-content/EN /TXT/PDF/?uri=CEL EX:32002H0413\&from=EN. Acesso em: 17 de nov. de 2016.

CPRM - SERVIÇO GEOLÓGICO DO BRASIL. Projeto cadastro de fontes de abastecimento por água subterrânea estado de Pernambuco: diagnóstico do município de Ilha de Itamaracá. Recife: CPRM/PRODEEM, 2005.

FABBRI, K. P. A methodology for supporting decision making in integrated coastal zone management. Ocean \& Coastal Management, v. 39, p. 51-62, 1998.

IBGE - Instituto Brasileiro de Geografia e Estatística. Censo demográfico 2010. Características da população e dos domicílios. Resultados do universo. Rio de Janeiro-RJ: IBGE, 2010.

MISDORP, R. What is ICZM? Basic elements of coastal cooperation, p.126-129, 2011. Disponível em: http://www.coastal cooperation.net/part-III/III-1.pdf. Acesso em: 17 de nov. de 2016.

OLSEN, S.; TOBEY, J.; KERR, M. A common framework for learning from ICM experience. Ocean \& Coastal Management, v. 37, p. 155-174, 1997.

PINHO, L.; MARTINS, F.; ALMEIDA, A.CASTRO, R. E. F.; MELO, M. H. S.; SILVARES, E. F. M. Socio-territorial GIS as a tool to support Coastal Management. Proceedings: CoastGIS 07: The 8th International Symposium on GIS and Computer Mapping for Coastal Zone Management, 8, 2007, Aveiro: University of Cantabria, p. 87-96, 2007.

PODVEZKO, V. Application of AHP technique. Journal of Busi ness Economics and Management, v.10, n.2, p.181-189, 2011.

POST, J. C. \& LUNDIN, C. G., Guidelines for integrated coastal zone management. Environmentally Sustainable Development Studies and Monographs Series. n. 9. The World Bank, Washington, DC, 16 p., 1996.

SAATY, T. L. The Analytic Hierarchy Process: Planning, Priority Setting, Resource Allocation, $2^{\mathrm{a}}$ ed. Pittsburgh: RW Publications, 287 p., 1990.

SCHMIDT, M. A. R.; BARBOSA, G. R. Uso de redes neurais artificiais na ponderação inicial da técnica AHP em análises de vulnerabilidade de bacias hidrográficas. Boletim de Ciências Geodésicas, v. 22, p. 511-525, 2016.

Submetido em 17 de novembro de 2016 Aceito em $1^{\circ}$ de fevereiro de 2017 